\title{
Spontaneous resolution of serous retinal detachment caused by choroidal mass after a first trimester abortion
}

\author{
You Hyun Lee, Yu Cheol Kim \\ Department of Ophthalmology, Keimyung University Dongsan Hospital, Keimyung University School of Medicine, Daegu, Korea
}

Received: February 27, 2020

Revised: March 9, 2020

Accepted: March 11, 2020

Corresponding author:

Yu Cheol Kim

Department of Ophthalmology,

Keimyung University Dongsan

Hospital, Keimyung University

School of Medicine, 1035

Dalgubeoldae-ro, Dalseo-gu,

Daegu 42601, Korea

Tel: +82-53-258-4545

Fax: +82-53-258-7130

E-mail: eyedr@dsmc.or.kr
Pregnancy-related ocular diseases develop mostly in the third trimester of pregnancy. Here, we describe a case of a pregnant woman with a choroidal mass that caused a serous retinal detachment during the first trimester of pregnancy. The patient's condition resolved spontaneously after an abortion.

Keywords: Choroidal neoplasm; Choroidal osteoma; First trimester; Pregnancy

\section{Introduction}

During pregnancy, cardiac output increases and hormone levels fluctuate. These physiological changes in pregnant women often cause retinal and choroidal diseases or worsen preexisting retinal and choroidal conditions $[1,2]$. For example, the incidence of central serous chorioretinopathy (CSC) and circumscribed choroidal hemangioma $(\mathrm{CCH})$ increase during pregnancy $[3,4]$. Specifically, these diseases become exacerbated during the third trimester and spontaneously regress after childbirth $[5,6]$. Here, we present a case of a pregnant woman with a choroidal mass causing serous retinal detachment (SRD) during the first trimester of pregnancy that resolved spontaneously after an abortion.

\section{Case}

This study was approved by the Institutional Review Board of the Keimyung University Dongsan Hospital (IRB No: DSMC 202002-070). The patient provided written informed consent for pub- lication of clinical details and images.

A 40-year-old woman was referred to our hospital with a 3-day history of visual disturbance in her left eye. The patient was a primigravida who was in her first trimester of pregnancy ( 7 weeks) at the time of referral. She did not have gestational hypertension or diabetes. Her left eye initially had a best-corrected visual acuity (BCVA) of 20/50 (according to the Snellen chart) and an intraocular pressure (IOP) of $15 \mathrm{mmHg}$. Her right eye had a BCVA of 20/20 and an IOP of $17 \mathrm{mmHg}$. Examination of the fundus revealed an orange-red retina in the left eye with posterior pole elevation (Fig. 1A).

Optical coherence tomography (OCT; DRI OCT Triton, Topcon, Tokyo, Japan) revealed a thick ( $>500 \mu \mathrm{m}$ ) elevated choroid, an SRD, compression of the choriocapillaris, and a choroidal mass located between the choriocapillaris and the outer choroidal tissue in the left eye (Fig. 1B). We avoided fluorescein angiography (FA) and indocyanine green angiography (ICGA) because of their possible teratogenicity. The patient was diagnosed with a choroidal mass. The appearance of the mass suggested possible

Copyright (C) 2020 Yeungnam University College of Medicine

This is an Open Access article distributed under the terms of the Creative Commons Attribution Non-Commercial License (http://creativecommons.org/licenses/by-nc/4.0/) which permits unrestricted non-commercial use, distribution, and reproduction in any medium, provided the original work is properly cited. 
$\mathrm{CCH}$. We decided to closely monitor the changes in the patient's BCVA and the progression of her SRD.

One month later, the patient revisited our clinic after having had an abortion during her 9th week of pregnancy. Her left-eye BCVA was 20/20 and her IOP was $14 \mathrm{mmHg}$. Examination of the fundus showed that the elevated retinal height had decreased and that the retinal surface exhibited a patchy yellowish discoloration (Fig. 2A). OCT revealed a decreased SRD with visible fine lamellar lines of the choroidal lesion (Fig. 2B). FA (HRA-2; Heidelberg Engineering, Dossenheim, Germany) revealed patchy hyperfluorescence in the area corresponding with the previously elevated retina during the arteriovenous phase (Fig. 3A) with latephase staining (Fig. 3B). ICGA revealed early hypofluorescence
(Fig. 3C), followed by diffuse confluent fluorescence during the late phase (Fig. 3D). We requested that the patient visit the hospital 3 months later, however she was lost during this follow-up period.

\section{Discussion}

$\mathrm{CCH}$ appears as an indistinct, round-to-oval, orange-red mass located posterior to the equator of the eye [6]. CSC is characterized as an accumulation of subretinal fluid that causes circumscribed neurosensory retinal detachment [7]. Distinguishing between these diseases during pregnancy is difficult because using either FA or ICGA is discouraged during this period owing to their possible teratogenicity [5]. In this case, at 7 weeks of pregnancy, we
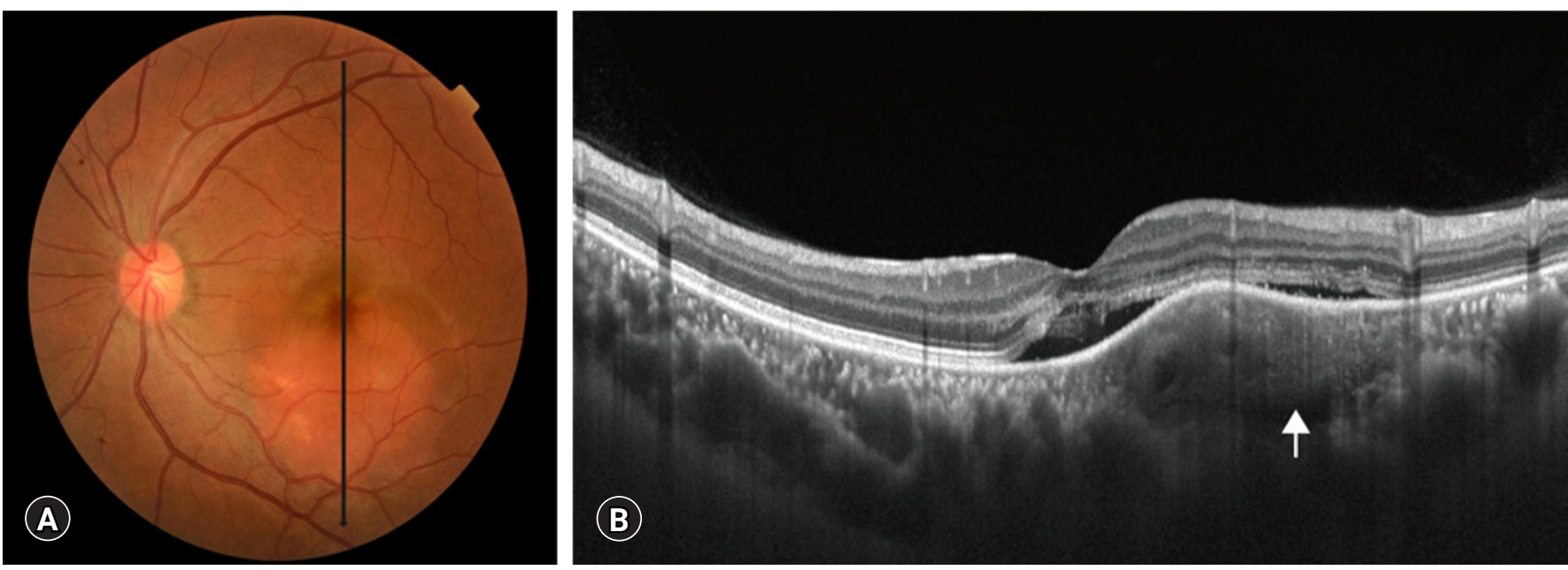

Fig. 1. Fundus photograph and optical coherence tomography of the left eye obtained on the initial visit. (A) An elevated orange-red retina is visible at the posterior pole. (B) Optical coherence tomography reveals a thick choroid, compression of the choriocapillaris, and serous retinal detachment with a choroidal mass (arrow).
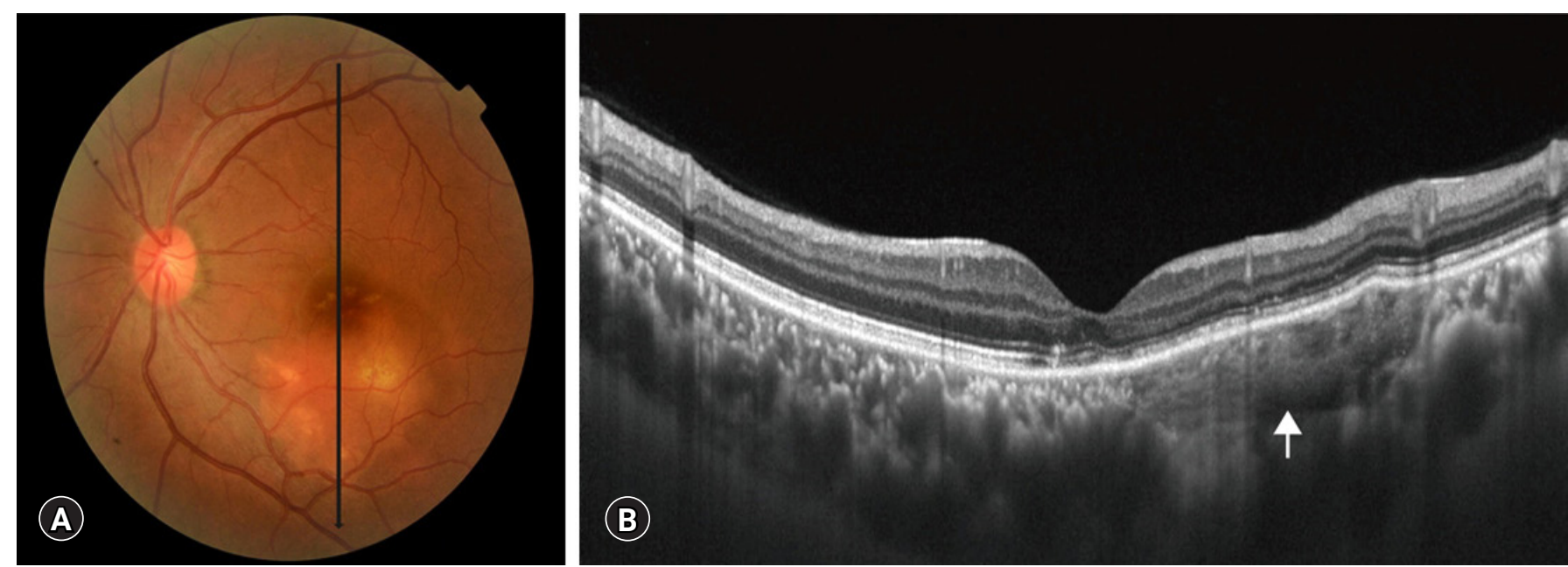

Fig. 2. Fundus photograph and optical coherence tomography of the left eye obtained 1 month after the initial visit. (A) The height of the elevated retina is reduced and patchy yellowish discoloration is seen. (B) The serous retinal detachment is resolved and fine lamellar lines within the choroidal lesion are visible (arrow). 

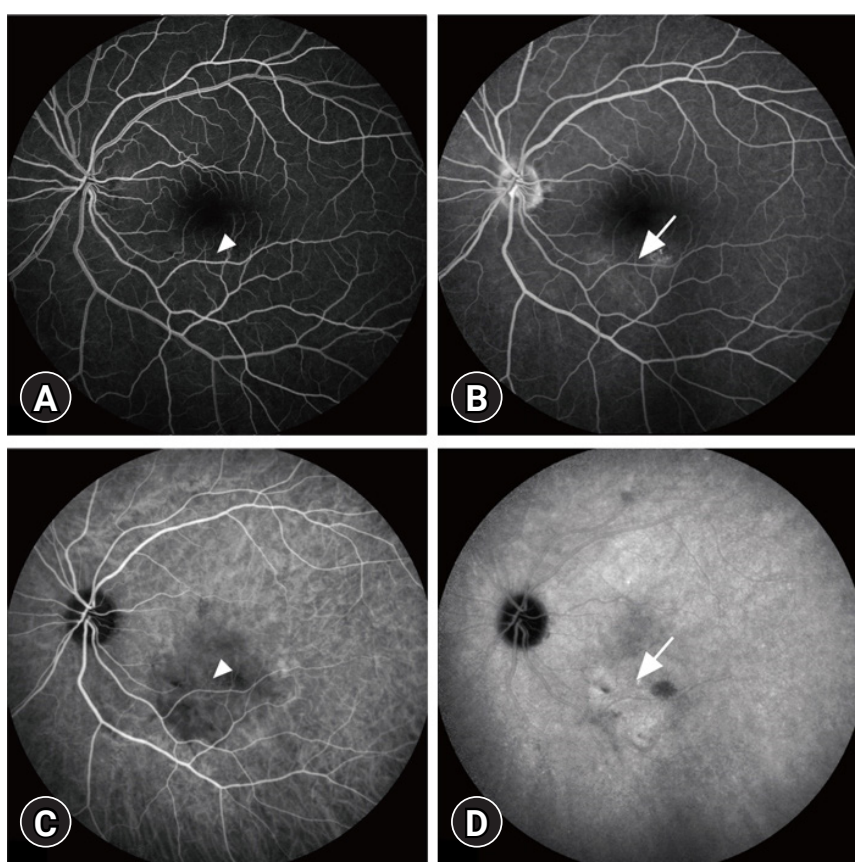

Fig. 3. Fluorescein angiography and indocyanine green angiography of the left eye obtained 1 month after the initial visit. Fluorescein angiography ( $A$, arteriovenous phase; $B$, late phase) reveals early hyperfluorescence (arrowhead) with latephase staining (arrow). Indocyanine green angiography $(C$, early phase; $D$, late phase) reveals early hypofluorescnece (arrowhead) followed by diffuse confluent fluorescence in the late phase (arrow).

initially suspected $\mathrm{CCH}$ because of the lesion's orange-red elevated appearance, the presence of SRD, and the presence of a thick and elevated choroid with an associated mass. Additionally, we eliminated the possibility of CSC as it typically develops in women with pre-eclampsia [8]. FA and ICGA were performed after the patient's abortion. FA revealed early patchy hyperfluorescence with late-phase staining, and ICGA revealed early hypofluorescence with later diffuse confluent fluorescence. The FA findings are consistent with the $\mathrm{CCH}$ diagnosis, as $\mathrm{CCH}$ is characterized by a stippled choroidal hyperfluorescence followed by increasing hyperfluorescence and progressive staining of the tumor. However, these FA characteristics are not pathognomonic for $\mathrm{CCH}$. Unlike the FA findings, the ICGA findings are inconsistent with the $\mathrm{CCH}$ diagnosis, as $\mathrm{CCH}$ manifests as extreme early hyperfluorescence that decreases in the later phases (known as the "washout" phenomenon) [9]. Compression of the choriocapillaris was visible upon the initial OCT, however $\mathrm{CCH}$ typically develops in the absence of choriocapillaris compression [10].

Choroidal osteomas are benign, rare, ossifying choroidal tumors that typically affect the juxtapapillary and macular areas of young healthy females [11]. The color of the choroidal osteoma depends on the depigmentation of the retinal pigment epithelium [12]. In our case, an orange-red lesion in the fundus was seen at an early stage, which is in line with the diagnostic features of $\mathrm{CCH}$. The fine lamellar lines found within the choroidal mass during OCT after the patient's abortion indicated the possible presence of an early choroidal osteoma. Moreover, ICGA of choroidal osteoma usually reveals early hypofluorescence and latephase hyperfluorescence [13]. However, we did not perform B-scan ultrasonography in this case, which was consistent with a previous study [14]. After the patient's abortion her decreased visual acuity improved and her SRD resolved, which implies that her pregnancy was directly related to the growth of the choroidal mass. While the mechanism remains unclear, we postulated that the increase in maternal cardiac output during the first trimester may have caused hyperpermeability of the vascular channels within the choroidal mass, which may have led to its exaggerated appearance [15].

In conclusion, it is possible for SRD to develop in first trimester of pregnancy along with an associated choroidal mass. This SRD will most likely resolve spontaneously after an abortion or the termination of pregnancy. Our finding highlights the direct correlation between pregnancy and choroidal masses, which may help physicians and specialists anticipate these issues in pregnant patients.

\section{Acknowledgments}

\section{Conflicts of interest}

No potential conflict of interest relevant to this article was reported.

\section{Funding}

This work was supported by the National Research Foundation of Korea grant (NRF-2019R1G1A1011559).

\section{Author contributions}

Conceptualization: YHL, YCK; Data curation: YHL; Formal analysis: YCK; Supervision: YCK; Writing-original draft: YHL; Writing-review \& editing: YCK.

\section{ORCID}

You Hyun Lee, https://orcid.org/0000-0001-8116-7942

Yu Cheol Kim, https://orcid.org/0000-0003-1615-6651

\section{References}

1. Thornburg KL, Jacobson SL, Giraud GD, Morton MJ. Hemo- 
dynamic changes in pregnancy. Semin Perinatol 2000;24:11-4.

2. Errera MH, Kohly RP, da Cruz L. Pregnancy-associated retinal diseases and their management. Surv Ophthalmol 2013;58: 127-42.

3. Chatziralli I, Kabanarou SA, Parikakis E, Chatzirallis A, Xirou T, Mitropoulos P. Risk factors for central serous chorioretinopathy: multivariate approach in a case-control study. Curr Eye Res 2017;42:1069-73.

4. Cohen VM, Rundle PA, Rennie IG. Choroidal hemangiomas with exudative retinal detachments during pregnancy. Arch Ophthalmol 2002;120:862-4.

5. Sayman Muslubas I, Arf S, Hocaoglu M, Ozdemir H, Karacorlu M. Spontaneous regression of serous retinal detachment associated with circumscribed choroidal hemangioma after childbirth. Retin Cases Brief Rep 2017;11:7-11.

6. Heimann H, Damato B. Congenital vascular malformations of the retina and choroid. Eye (Lond) 2010;24:459-67.

7. Daruich A, Matet A, Dirani A, Bousquet E, Zhao M, Farman N, et al. Central serous chorioretinopathy: recent findings and new physiopathology hypothesis. Prog Retin Eye Res 2015;48:82118.

8. Morikawa M, Cho K, Kojima T, Chiba K, Ishikawa S, Umazume T, et al. Risk factors for central serous chorioretinopathy in pregnant Japanese women. J Obstet Gynaecol Res 2017;
43:866-72.

9. Arevalo JF, Shields CL, Shields JA, Hykin PG, De Potter P. Circumscribed choroidal hemangioma: characteristic features with indocyanine green videoangiography. Ophthalmology 2000; 107:344-50.

10. Rojanaporn D, Kaliki S, Ferenczy SR, Shields CL. Enhanced depth imaging optical coherence tomography of circumscribed choroidal hemangioma in 10 consecutive cases. Middle East Afr J Ophthalmol 2015;22:192-7.

11. Shields CL, Shields JA, Augsburger JJ. Choroidal osteoma. Surv Ophthalmol 1988;33:17-27.

12. Gass JD, Guerry RK, Jack RL, Harris G. Choroidal osteoma. Arch Ophthalmol 1978;96:428-35.

13. Lafaut BA, Mestdagh C, Kohno T, Gaudric A, De Laey JJ. Indocyanine green angiography in choroidal osteoma. Graefes Arch Clin Exp Ophthalmol 1997;235:330-7.

14. Hussain R, Anantharaman G, Rajesh B, Gopalakrishnan M. Real-time in vivo micromorphology and histopathology of choroidal osteoma using enhanced depth imaging. Indian J Ophthalmol 2015;63:453-5.

15. Robson SC, Hunter S, Boys RJ, Dunlop W. Serial study of factors influencing changes in cardiac output during human pregnancy. Am J Physiol 1989;256(4 Pt 2):H1060-5. 